\title{
Towards 'Interactive' Active Learning in Multi-view Feature Sets for Information Extraction
}

\author{
Katharina Probst and Rayid Ghani \\ Accenture Technology Labs, Chicago, IL, USA \\ katharina.a.probstaccenture.com, rayid.ghani@accenture.com
}

\begin{abstract}
Research in multi-view active learning has typically focused on algorithms for selecting the next example to label. This is often at the cost of lengthy wait-times for the user between each query iteration. We deal with a real-world information extraction task, extracting attribute-value pairs from product descriptions, where the learning system needs to be interactive and the users time needs to be used efficiently. The first step uses coEM with naive Bayes as the semi-supervised algorithm. This paper focuses on the second step which is an interactive active learning phase. We present an approximation to coEM with naive Bayes that can incorporate user feedback almost instantly and can use any sample-selection strategy for active learning. Our experimental results show high levels of accuracy while being orders of magnitude faster than using the standard coEM with naive Bayes, making our IE system practical by optimizing user time.
\end{abstract}

\section{Introduction}

There has been a lot of recent research in semi-supervised learning algorithms for multiview feature sets that deal with learning problems where each example is described by two distinct sets of features, either of which is sufficient to approximate the function; that is, they fit the cotraining problem setting [13|8]. These approaches have been applied to several domains such as Named Entity Recognition, Information Extraction, Text Classification, and Speech Recognition. The major motivation behind this work has been to exploit the multiple feature sets in order to reduce the number of labeled examples required to learn by making use of the abundance and cheapness of unlabeled data.

Active learning is a closely related field of research that seeks to make efficient use of a trainer's time by intelligently selecting examples to label based on the anticipated value of that label to the learner. Because of the effectiveness of multi-view semi-supervised algorithms, several techniques have been proposed to combine semisupervised learning with active learning. [7] proposed Co-EMT which interleaves semisupervised coEM (with naive Bayes) and co-Testing. [6] propose active learning with multiple feature sets and experiment with various selection strategies. Both of these contributions show the efficacy of using unlabeled data by combining multi-view semisupervised learning with active learning.

However, the focus, as in most other active learning research, has mostly been on improving algorithms and measures for selecting the next example to be labeled by the user. This has often been at the cost of increased time between iterations of active 
learning feedback which in practice results in lengthy wait times between interactions for the user. Here, we address the 'interactive' aspect of active learning which is one of the major motivations for this line of research - making the most efficient use of the trainer's time. Our motivation comes from a real-world information extraction task: extracting attribute-value pairs from free-text product descriptions [9]. The work presented in this paper aims at making the process of extracting attribute-value pairs from product descriptions more efficient and cheaper by developing an interactive tool.

Our system consists of two parts. The first part is a semi-supervised (non-interactive) classifier (coEM with naive Bayes) that results in a set of attribute-value pairs described in earlier work [9]. The focus of this paper is the next step where the user works with our tool to provide feedback in order to improve the extraction accuracy. When coEM is interleaved in the active learning phase as the underlying semi-supervised classifier (as in [7], [6]), we get good extraction accuracy but the wait time for the user becomes extremely long. In order to make this active learning phase faster while achieving comparable accuracy, we developed a fast approximation to coEM with naive Bayes that can incorporate user feedback almost instantly and can work with any sample-selection strategy for active learning. Our experimental results, on a real world data set of sports products, show high levels of accuracy while being orders of magnitude faster than using the standard coEM with naive Bayes, making our system practical by minimizing user wait time between feedback iterations.

\section{Attribute Extraction}

Before we discuss our contributions in active learning, we briefly describe the larger system we are building. Our goal is to take natural language product descriptions (such as those on retail catalogs or websites) and extract sets of attribute-value pairs. The products can then be represented in a structured way, which enables a number of business applications, e.g., product recommendations, assortment optimization, and pricing.Since there are no off-the-shelf tools available to extract these attribute-value pairs, it is mostly being done manually which makes the process very expensive. Our system automates the extraction and then learns incrementally from user feedback.

\section{Making coEM with Naive Bayes Fast}

In this paper, we focus on developing a fast semi-supervised learning algorithm in the context of an active learning system that is interactive. Since the semi-supervised learning algorithm needs to be retrained at every iteration of active learning, it commonly leads to long spans when the user sits idle waiting for the system, something we want to avoid.

Our main contribution is a fast approximation to coEM with naive Bayes which is used in active learning in order to make our system interactive. The sections below describe coEM with naive Bayes (our initial approach which was accurate but slow), an approximation to coEM with naive Bayes, and the fast version of that approximation.

Our initial system uses coEM with naive Bayes since that has been previously shown to outperform other semi-supervised algorithms for text learning and information tasks 
[45]7]. When we incorporate this algorithm in our active learning setting, we get high extraction accuracy but the training time at each iteration is too slow. Thus, we developed an approximation that uses relative frequencies and devise a fast version of it by formulating coEM as a matrix multiplication algorithm. This fast approximation to coEM with naive Bayes is able to retrain and incorporate new labeled data instantly, making our attribute extraction system practical by minimizing user wait time. The sections below describe each of these three algorithms: coEM with naive Bayes (our initial approach which was accurate but slow), approximation to coEM with naive Bayes, and the fast version of that approximation.

\section{1 coEM}

For the initial (batch) learning phase, our system uses coEM [8] which has been shown to outperform other semi-supervised algorithms for several text learning tasks [4 [5]7].

To express the data in two views, each word is expressed in viewl by the stemmed word itself, plus the part of speech as assigned by the Brill tagger [2]. The view2 for this data item is a context of window size 8, i.e. up to 4 words (plus parts of speech) before and up to 4 words (plus parts of speech) after the word or phrase in view1. We are currently experimenting with adding additional features (such as syntactic features, spelling features, etc.) to measure whether they can significantly enhance performance. By default, all words are processed into viewl as single words. Sequences of words with high correlation scores (Yule's Q, $\chi^{2}$, and pointwise mutual information) are treated as phrasal entities and thus as a single view1 data item. Co-EM uses the initial labels to label all training examples in view1. These labels are then used to probabilistically label all view2 elements [9]. The view2 labels are in turn used to relabel view1, etc.

\subsection{Simpler Version of Co-EM with Naive Bayes}

When we use coEM with naive Bayes as the semi-supervised algorithm in our active learning setting, the wait time between interactions is unacceptable 1 . To make our tool interactive, we propose an approximation to coEM with naive Bayes that does not compromise much on accuracy but is orders of magnitude faster. We briefly describe the algorithm here, and give the fast version of it in the next section. The algorithm works as follows (following [5]): Suppose we want to determine a distribution over all possible labels for each training element, both in view1 and view2. In our case, the possible labels are unassigned (the default before learning), attribute, value, neither (all items default to unassigned at the beginning). Denote these labels as $p(1 \mid\langle$ view 1 , view 2$\rangle)$, $\ldots, p(4 \mid\langle$ view 1 , view 2$\rangle)$. The goal of the algorithm is to assign these probabilities to each item. In each iteration, however, we label view1 and view2 separately as follows. Using the initial labeled data, we assign all labeled view1 items to their respective class (attribute, value, or neither) as the initial labeling. The next step is to relabel all view2 data items using the view1 labels. The label distribution of a view2 element $v 2_{i 2}, 1 \leq i 2 \leq n 2$, where $n 2$ is the number of distinct $v 2$ elements, is obtained from the view 1 elements $v 1_{i 1}, 1 \leq i 1 \leq n 1$, where $n 1$ is the number of distinct $v 1$ elements,

\footnotetext{
${ }^{1}$ See section 4 for more details.
} 
it aligns with, weighted by the number of times the $v 1$ and $v 2$ elements align. Denote $\operatorname{cooc}\left(v 1_{i 1}, v 2_{i 2}\right)$ as the number of times $v 1_{i 1}$ and $v 2_{i 2}$ align to each other. Then:

$$
p\left(1 \mid v 2_{i 2}\right)=\frac{\sum_{i 1=1}^{n 1} \operatorname{cooc}\left(v 2_{i 2}, v 1_{i 1}\right) * p\left(1 \mid v 1_{i 1}\right)}{\sum_{i 1=1}^{n 1} \operatorname{cooc}\left(v 2_{i 2}, v 1_{i 1}\right)}
$$

Similarly for $p\left(2 \mid v 2_{i 2}\right), p\left(3 \mid v 2_{i 2}\right)$, and $p\left(4 \mid v 2_{i 2}\right)$. Re-estimation of all view1 elements follows in the reverse way. However, for those $v 1_{i 1}$ that are found in the initial labels, there is no re-estimation.The re-estimation steps are repeated until convergence or for a fixed number of iterations. The final probabilities for a data item in its context is finally assigned by averaging $p\left(j \mid v 1_{i 1}\right)$ and $p\left(j \mid v 2_{i 2}\right)$ for $1 \leq j \leq 4$.

\subsection{Fast Incremental Approximation of coEM with Naive Bayes}

We will now reformulate a fast, incremental variation of the above algorithm that compromises little on accuracy but is orders of magnitude faster. The first insight is that the re-estimation can essentially be viewed as a matrix multiplication: Let $A_{1}$ be the probability matrix of view1 that is obtained by the initial labeling of all view1 elements using the labeled data. $A_{1}$ is of size $n_{1} \times 4$. Denote each view1 data element as $v 1_{i}, 1 \leq i \leq n 1$, where $n 1$ is the number of view 1 data elements. Denote the classes $c_{j}, 1 \leq j \leq 4$, where the classes are as described above. This matrix will have the following form:

$$
A_{1}=\left[\begin{array}{cccc}
p\left(c_{1} \mid v 1_{1}\right) & p\left(c_{2} \mid v 1_{1}\right) & p\left(c_{3} \mid v 1_{1}\right) & p\left(c_{4} \mid v 1_{1}\right) \\
p\left(c_{1} \mid v 1_{2}\right) & p\left(c_{2} \mid v 1_{2}\right) & p\left(c_{3} \mid v 1_{2}\right) & p\left(c_{4} \mid v 1_{2}\right) \\
\ldots & \ldots & \ldots & \ldots \\
p\left(c_{1} \mid v 1_{n 1}\right) & p\left(c_{2} \mid v 1_{n 1}\right) & p\left(c_{3} \mid v 1_{n 1}\right) & p\left(c_{4} \mid v 1_{n 1}\right)
\end{array}\right]
$$

Let $A_{2}$ be the $n_{2} \times 4$ probability matrix of view2, defined in the same ways as $A_{1}$. Further, let $B_{1,2}$ be the $\left(n_{1} \mathrm{x} n_{2}\right)$ transition matrix from view1 to view2. This transition matrix is a sparse matrix that stores for every view 1 entry all the view 2 data elements that it co-occurs with, along with the count of how often the view1 and view2 co-occur. The transition matrices are normalized by the total number of co-occurrences for each data element. This matrix will take the following form:

$$
\begin{gathered}
B_{1,2}=\left[\begin{array}{cccc}
p\left(v 2_{1} \mid v 1_{1}\right) & p\left(v 2_{2} \mid v 1_{1}\right) & \ldots & p\left(v 2_{n 2} \mid v 1_{1}\right) \\
p\left(v 2_{1} \mid v 1_{2}\right) & p\left(v 2_{2} \mid v 1_{2}\right) & \ldots & p\left(v 2_{n 2} \mid v 1_{2}\right) \\
\ldots & \ldots & \ldots & \ldots \\
p\left(v 2_{1} \mid v 1_{n 1}\right) & p\left(v 2_{2} \mid v 1_{n 1}\right) \ldots & p\left(v 2_{n 2} \mid v 1_{n 1}\right)
\end{array}\right] \text { Where } \\
p\left(v 2_{i 2} \mid v 1_{i 1}\right)=\frac{\operatorname{cooc}\left(v 2_{i 2}, v 1_{i 1}\right)}{\sum_{i 2=1}^{n 2} \operatorname{cooc}\left(v 2_{i 2}, v 1_{i 1}\right)} .
\end{gathered}
$$


$B_{2,1}$ is defined in an analogous way. Each iteration of the coEM algorithm can then be seen as a matrix multiplication:

$$
A_{2}^{\prime}=B_{2,1} * A_{1} \text {, Similarly: } A_{1}^{\prime}=B_{1,2} * A_{2}^{\prime}
$$

This multiplication is equivalent to the above iterative algorithm for those items that are not in the initial training data: each cell $(i, j)$ in the resulting matrix will be the result of the sum of all the probabilities for column $j$ (the class label) for all the data items in the other view with which $i$ has a non-zero transition probability, weighted by this transition probability. Note also that these multiplications are the first iteration of the coEM algorithm. Further iterations proceed by the same principle, e.g., $A_{2}^{\prime \prime}=B_{2,1} * A_{1}^{\prime}$. This computation is recursive, and the following holds:

$$
\begin{aligned}
A_{2}^{\prime \prime} & =B_{2,1} * A_{1}^{\prime}=B_{2,1} *\left(B_{1,2} * A_{2}^{\prime}\right) \\
& =B_{2,1} *\left(B_{1,2} *\left(B_{2,1} * A_{1}\right)\right)=\left(B_{2,1} * B_{1,2} * B_{2,1}\right) * A_{1}
\end{aligned}
$$

Similarly for further iterations. The modified probability matrix of each view is computed by multiplying the original probability matrix of the other view by a product of transition matrices, where the number of factors is a function of the number of desired coEM iterations. Thus, coEM can either be run iteratively as described in the previous section, or by multiplying the original $A_{1}$ by the transition matrices.

When recomputing $A_{2}$, we will have a product of transition matrices that is different from the one used for recomputing $A_{1}: A_{1}$ will be recomputed as follows:

$$
A_{1}^{n}=\left(B_{1,2} * \cdots * B_{1,2} * B_{2,1}\right) * A_{1}=T_{1,1} * A_{1}
$$

whereas $A_{2}$ will be recomputed with:

$$
A_{2}^{n}=\left(B_{2,1} * \cdots * B_{1,2} * B_{2,1}\right) * A_{1}=T_{2,1} * A_{1}
$$

$T_{1,1}$ and $T_{2,1}$ are the products of transition probabilities. Their interpretation is as follows: each cell $(i, j)$ in the matrix $T_{1,1}$ represents the influence view1 data element $j$ has on view 1 data element $i$ after $n$ iterations; similarly for $T_{2,1}$.

So far, we have described the base algorithm, and how it can be expressed as a set of matrix multiplications. However, as laid out in the iterative algorithm, re-estimation as described here only applies to the cases where a data item is not already known from the original labeled data. For known examples, the iterative algorithm will proceed by simply not relabeling view 1 elements in any of the iterations. The computation of $T_{1,1}$ and $T_{2,1}$ as described above did not take this into account. Rather, in such cases, the transition matrix should reflect that the view1 element is known. To see how this can be accomplished, note again that the transition matrices capture how much impact one data element has on another. Known data items receive the same probability distribution in each iteration, i.e., they should not be updated from other data items. This is done by setting all transition probabilities in $T_{1,1}$ into the data item to 0 except the transition from itself, i.e., the row for data item $v 1_{i 1}$ is all 0 s except for the cell $T_{1,1}(i 1, i 1)$.

Although we have now reformulated the coEM algorithm, we have not yet dealt with user feedback. How can this be done efficiently? Note that new labeled examples do not modify the transition matrix, but rather only the current probability matrix, i.e., $A_{1}$. This is because the transition matrix only captures the cooccurrence counts, and says nothing 
about the labels for either the view1 or the view2 element. For this reason, a user interaction will not have any impact on the transition matrix products $T_{1,1}$ and $T_{2,1}$, and we can therefore precompute the transition matrix products. The interactive algorithm will now proceed as follows: in an offline step, we precompute the matrices $T_{1,1}$ and $T_{2,1}$. When the user provides feedback, we simply modify $A_{1}$, recompute $A_{1}$ and $A_{2}$ by multiplication with the transition matrices. Final probabilities are then assigned as in the iterative algorithm (i.e., by averaging the view1 and view2 probabilities).

\section{Experimental Results}

For the experiments reported in this paper, we crawled the web site of a sporting goods retailet 2 , concentrating on the domain of tennis. The crawler gives us a set of product descriptions, which we use as unlabeled training data. Some examples are:

\section{4 rolls white athletic tape \\ Audio/Video Input Jack \\ Vulcanized latex outsole construction is lightweight and flexible}

For more details on the dataset, please refer to [9]. The experiments reported here were run on 3194 product descriptions. The results were then compared to 620 randomly selected descriptions that were manually labeled with the correct pairs. Below, we show a sample of extracted attribute-value pairs:

\section{$\langle$ val $\rangle$ 1 1/2-inch $\langle/$ val $\rangle\langle$ att $\rangle$ polycotton blend tape $\langle/$ att $\rangle$ \\ $\langle$ val $\rangle 1 \mathrm{roll}\langle/$ val $\rangle\langle$ att $\rangle$ underwrap $\langle/$ att $\rangle$ \\ Synthetic $\langle$ val $\rangle$ leather $\langle$ val $\rangle\langle$ att $\rangle$ upper $\langle$ att $\rangle$ \\ $\langle$ val $\rangle$ adiWear tough $\langle/ v a l\rangle\langle$ att $\rangle$ rubber outsole $\langle$ att $\rangle$}

We provide results comparing the performance of the fast approximation to coEM with naive Bayes. The first set of results demonstrates that the approximation yields comparable performance to coEM with naive Bayes. We compare three sample selection methods over 25 user interaction iterations: the first method randomly selects the next example to present to the user. Another method, density-based sample selection, selects the most frequent data elements. Finally, KL-divergence is a method that selects those examples that the algorithm is most uncertain about. We measure uncertainty by the KL-divergence between the view1 and view2 probability distributions for each data element. The KL-divergences are weighted by frequency. For precision (figure 1a), we report only fully correct pairs, because almost all (around 96\%) of the extracted pairs are at least partially correct in the baseline system. We further report recall (figure 1b), i.e., how many of the manually extracted pairs were at least partially extracted by the system. Finally, we report the f-measure (figure 1c).

The results, as in previous active learning studies, show that density and KLdivergence sample selection outperform random sample selection consistently. More importantly to this paper, the results also show that the proposed algorithm is an effective approximation of the coEM algorithm with naive Bayes for our task. Moreover, this statement holds true for all the sample selection methods that are commonly used in this

${ }^{2}$ www.dickssportinggoods.com 
(a)

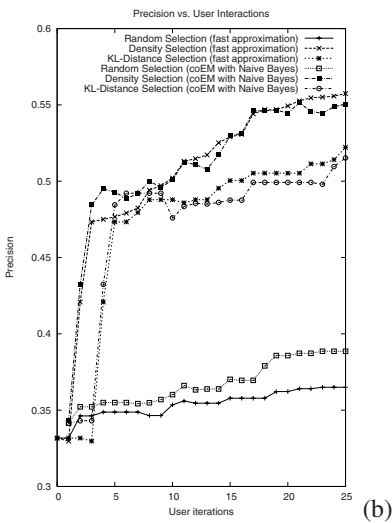

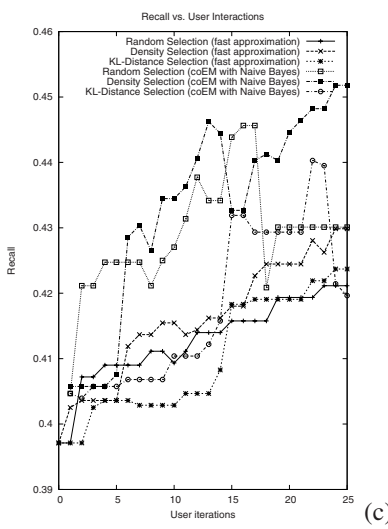

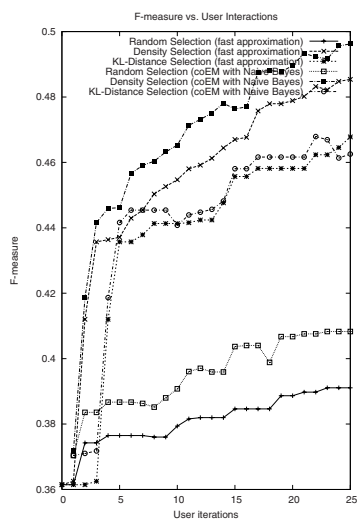

Fig. 1. Precision, Recall, and F-measure for fast algorithm compared to coEM with naive Bayes. The $\mathrm{y}$-value for $k$ indicates the recall, precision, and F-measure after the $k^{t h}$ user interaction

(a)

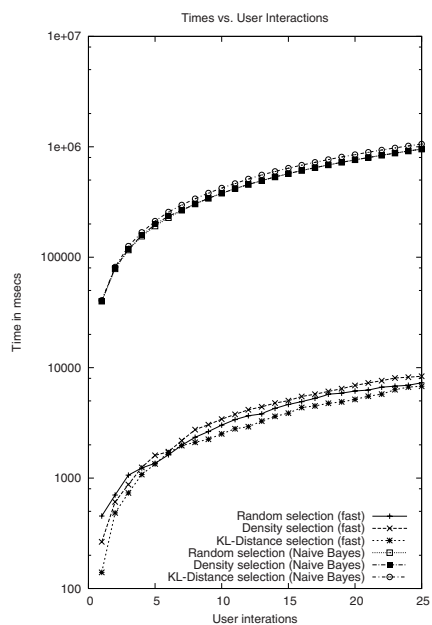

(b)

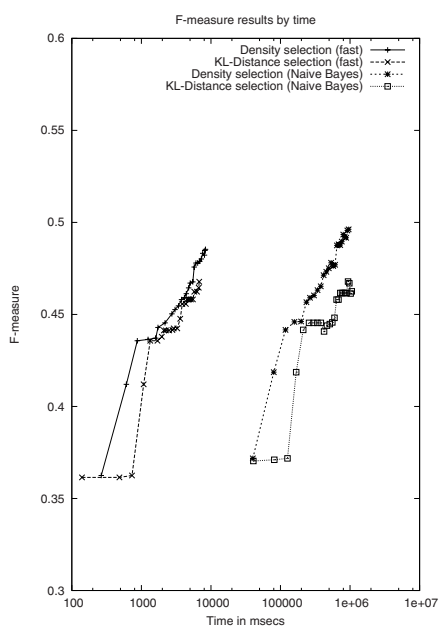

Fig. 2. Time comparison between Fast algorithm and coEM with naive Bayes. The y-value for $k$ indicates the time the user needed to wait until the $k^{\text {th }}$ user interaction.

setting. We then compare the fast approximation algorithm and coEM with naive Bayes in terms of user time. The time reported is the time that a user has to wait between interactions (iterations). We show that the fast approximation algorithm performs about two orders of magnitude faster than the coEM algorithm with naive Bayes (figure $2 \mathrm{a}$ ). The time is plotted on a log scale to enable plotting in one figure. We plot the cumulative times for user interactions. The results show that the interaction with the fast approximation algorithm is almost instantaneous, which was the goal of our work, whereas coEM with naive Bayes will require long user idle times. We finally combine the accuracy and time performance results to show that the fast approximation algorithm can 
yield comparable performance, but orders of magnitude faster (figure $2 b$ ). In fact, the performance after 25 user interactions was reached by the fast approximation algorithm before coEM with naive Bayes had finished even one user interaction iteration.

\section{Conclusions}

We addressed interactivity, an important issue that has not received much attention when combining active learning and semi-supervised learning for multi-view feature sets. Most research in multi-view active learning has focused on algorithms for selecting the next example to label. This is often at the cost of long wait-times for the user between each query iteration. We were motivated by a practical task, attribute-value extraction from product descriptions, where the learning system needs to be interactive and the user's time needs to be used efficiently. We use coEM with naive Bayes, a state-of-the art multi-view semi-supervised algorithm, which yields good extraction accuracy but is too slow to be interactives. We present an effective method for interactively incorporating user feedback in real-time with a fast approximation to coEM with naive Bayes where the bulk of computation can be done offline, before the user gets involved. Our approach can take any sample selection metric; we show experimental results with random, density-based and KL-divergence based metrics. Our experiments show that our algorithm gives comparable performance (precision, recall, and F1) to the original coEM with naive Bayes algorithm but is orders of magnitude faster. This results in our information extraction system being practical and minimizing user wait time. We believe that the combination of active learning and semi-supervised learning has enormous practical implications but the issue of long wait times can be a hinderance in many real applications. The work presented in this paper is a step towards building practical systems and will hopefully drive further research in this direction.

\section{References}

1. Blum, A., Mitchell, T.: Combining labeled and unlabeled data with co-training. In: COLT (1998)

2. Brill, E.: Transformation-based error-driven learning and natural language processing: A case study in part of speech tagging. Computational Linguistics (1995)

3. Collins, M., Singer, Y.: Unsupervised models for named entity classification. In: Joint SIGDAT Conference on EMNLP and VLC (1999)

4. Ghani, R., Jones, R.: A comparison of efficacy of bootstrapping algorithms for information extraction. In: LREC 2002 Workshop on Linguistic Knowledge Acquisition (2002)

5. Jones, R.: Learning to extract entities from labeled and unlabeled text. Ph.D. Thesis (2005)

6. Jones, R., Ghani, R., Mitchell, T., Riloff, E.: Active learning for information extraction with multiple view feature sets. In: Lavrač, N., Gamberger, D., Todorovski, L., Blockeel, H. (eds.) ECML 2003. LNCS (LNAI), vol. 2837, Springer, Heidelberg (2003)

7. Muslea, I., Minton, S., Knoblock, C.A.: Active + Semi-supervised Learning = Robust MultiView Learning. In: ICML (2002)

8. Nigam, K., Ghani, R.: Analyzing the effectiveness and applicability of co-training. In: CIKM (2000)

9. Probst, K., Ghani, R., Krema, M., Fano, A., Liu, Y.: Semi-supervised learning of attributevalue pairs from product descriptions. In: IJCAI (2007) 